\section{Re-evaluation of the classification system for the clinical interpretation of genomic copy number variation}

I agree with the opinion expressed by Wang and Chiang ${ }^{1}$ in the June issue of Genetics in Medicine that the categorical classification system implemented by ClinVar is in need of dramatic revision.

The complex ways in which multiple genes interact to cause disease are rapidly being revealed. A recent paper in the journal Science is a remarkable example of just such a case. These authors demonstrated that compound heterozygosity of three different genes causes childhood onset cardiomyopathy. ${ }^{2}$ Heterozygosity of any one or two was insufficient to cause the cardiac defect. Many of the most societally important conditions have genetic underpinnings that have yet to be fully elucidated. This is in large part because the genetics are non-Mendelian involving the action or misaction of more than a single gene. This emerging knowledge base needs to be reflected in the ClinVar Classification system. The classification of "pathological" or "benign" no longer reflects the current state of the science.

I would like to suggest an approach focused on the needs of the end user: the patient's treating physician. As geneticists we love to think about the molecular mechanism and mode of inheritance of disease. But for the treating physician the focus is on the probability of disease in their patient. The molecular mechanism is interesting to them only tangentially as it relates to providing optimal care to their patient.

In our own work to develop a tool to inform physicians about the gene-based clinical consequences of a chromosome 18 copy-number change we found that the gene function data led us to a classification system based on probability. ${ }^{3}$ Not surprisingly, the probability classifications also reflect the underlying molecular mechanism of disease and therefore are informative to the research community as well. Because we sought to describe the role of every gene on chromosome 18 with regard to dosage sensitivity, we were confronted with the entire spectrum of potential effects. This entire spectrum is not apparent in programs that cherry-pick only the welldescribed genes.

Because there are more data on the effects of hemizygosity than for suprazygosity, our gene dosage classifications for hemizygosity are better developed. In our system, the hemizygosity classification for the vast majority of genes is "no clinically significant effect." The group with the next- highest probability of being clinically relevant comprises the "risk factor" genes. These are genes that for example are found more often in people with autism than in people without. ${ }^{4}$ The next-highest risk classification is "conditional." These genes for the most part cause disease by a recessive mechanism. An abnormal phenotype is only apparent in the presence of second genetic event such as revealing a recessive variant on the remaining allele. Although this will be a low probability event, it is more probable than that of someone in the general population developing the disease. The next two classifications include the spectrum of probability for genes associated with haploinsufficiency. We found that several genes associated with disease only caused that disease in a low percentage of our cohort with hemizygosity for that gene. Clearly there are important discoveries to be made on the molecular mechanisms behind variable penetrance. We classified the genes associated with disease but with a penetrance less than $50 \%$ to be "low penetrance" genes. The next classification includes those genes that cause disease by haploinsufficiency but with a penetrance greater than $50 \%$. We also have the expectation that there will be some genes that are "haplolethal." However, we have yet to identify any on chromosome 18.

By creating a probability classification rather than dichotomous classifications the need to prove a degree of certainty before declaring a pathogenic finding is negated. By introducing probability as the discriminator it implies an inherent uncertainly and an exception of evolving knowledge. Rather than directing clinical decision-making it becomes a synthesis of current knowledge, much like OMIM or a review manuscript.

The difficulty in classifying genes as merely pathogenic or benign creates additional dilemmas. Some genes cause late childhood or adult onset conditions. Are these genes pathogenic or benign? Genes that cause treatable conditions such as myopia-are they pathogenic or benign? Not only are the dichotomous classifications insufficient they are misleading as well. The term "benign" implies that no action is necessary and "pathogenic" implies that action should be taken, when in fact neither conclusion may be prudent.

For a physician to learn that a gene has an $80 \%$ probability of causing a disease is more informative than knowing that the genetics community is $80 \%$ certain that a gene causes disease. They mean two very different things and may result in completely different approaches to clinical action and decision-making. Input from physicians who manage and treat people with genetic conditions as opposed to those who focus on the diagnosis of genetic conditions would be essential to creating a tool with maximum clinical utility. We offer our approach as an alternative way of defining the clinical effects of dosage sensitivity. 


\section{DISCLOSURE}

J.D.C. declares no conflicts of interest.

Publisher's note: Springer Nature remains neutral with regard to jurisdictional claims in published maps and institutional affiliations.

Jannine D. Cody, PhD (iD ${ }^{1,2}$

${ }^{1}$ Department of Pediatrics, University of Texas Health San Antonio, 7703 Floyd Curl Drive, San Antonio, TX 78229, USA; ${ }^{2}$ The Chromosome 18 Registry and Research Society, 7155 Oakridge Drive, San Antonio, TX 78229, USA. Correspondence: Jannine D. Cody (cody@uthscsa.edu)

\section{REFERENCES}

1. Wang NK, Chiang JPW. Increasing evidence of combinatory variant effects calls for revised classification of low penetrance alleles. Genet Med. 2019;21:1280-1282.

2. Gifford CA, Ranade SS, Samarakoon R, et al. Oligogenic inheritance of a human heart disease involving a genetic modifier. Science. 2019;364:865-870.

3. Cody JD, Heard P, Rupert D, et al. Chromosome 18 gene dosage map 2.0. Hum Genet. 2018;137:961-970.

4. Pinto D, Pagnamenta AT, Klei L, et al. Functional impact of global rare copy number variation in autism spectrum disorders. Nature. 2010;466:368-372

Advance online publication 9 August 2019. doi:10.1038/s41436-019-0619-6 